Int. J. Speleol. 5(1973), pp. 209-218.

\title{
Observations sur la faune aquatique souterraine de Cuba
}

\author{
par
}

\section{BOTOSANEANU*}

Ayant fait des prélèvements de faune souterraine aquatique pendant les expéditions biospéologiques cubano-roumaines de 1969 et 1973 à Cuba, je vais essayer de dégager ici quelques enseignements apportés par l'observation de cette faune, remarquable à plus d'un égard, ainsi que par celle de ses habitats.

D'assez nombreuses grottes de toutes les provinces de Cuba ont permis des récoltes de faune aquatique. Une particularité des grottes de Cuba (du karstostygal plus exactement) qui frappe l'esprit du chercheur, c'est - dans la zone vadose ou de percolation temporaire - le grand nombre de gours remplis par une pâte noirâtre constituée surtout de guano de Chauves-souris; pendant la saison sèche ce dépôt pâteux est surmonté par une couche d'eau douce de percolation ou de condensation (à $20,3-27^{\circ} \mathrm{C}$ ), dont l'épaisseur varie généralement de quelques $\mathrm{mm}$ à quelques $\mathrm{cm}$ seulement, bien que des quantités d'eau plus importantes aient pu être observées dans certains cas. C'est l'habitat d'une faunule extrêmement dense, constituée essentiellement d'Oligochètes, d'Ostracodes, de larves de Diptères (Psychodides etc.) auxquels s'ajoutent des Nématodes, des Rhabdocèles etc. Cette association peut ne pas être très intéressante du point de vue strictement faunistique, mais on doit souligner l'intérêt que présente l'existence, sous les tropiques, de cette "association d'hydro-guanobies" que n'a pas son équivalent dans les zones tempérées. Ainsi, la présence d'énormes colonies de Chauves-souris et de dépôts fort importants de guano, caractéristiques des zones tropicales, peut influer sur la nature des habitats aquatiques aussi.

Par contre, les gours sans dépôts de guano n'hébergent aucun des éléments de grande taille que nous venons de mentionner : leur faune est microscopique. Ajoutons que des animaux vivent, à Cuba comme en Europe, même dans les plus infimes bassins remplis d'eau de percolation : dans les "microgours", dans les alvéoles des "têtes" de stalagmites, nous avons pu constater par exemple - tout comme dans les grands gours tapissés de calcite cristallisée - la présence de nombreux Harpacticoïdes appartenant à des genres divers : Nitocrella, Canthosella, Elaphoidella, Parastenocaris (assez grand nombre d'espèces décrites par T. Petkovski).

Les cours d'eau souterrains permanents sont relativement rares à Cuba, ceux intermittents sont plus nombreux. Quelques éléments du plus haut intérêt ont pu être capturés dans des ruisseaux souterrains et surtout dans les accumulations d'eau laissées par ceux-ci sur la plancher des grottes actives, en période de sécheresse;

\footnotetext{
* Institut de Speologie E.G. Racovitza, 8 Rue Dr. Capsa, BUCURESTI 35, Roumanie.
} 


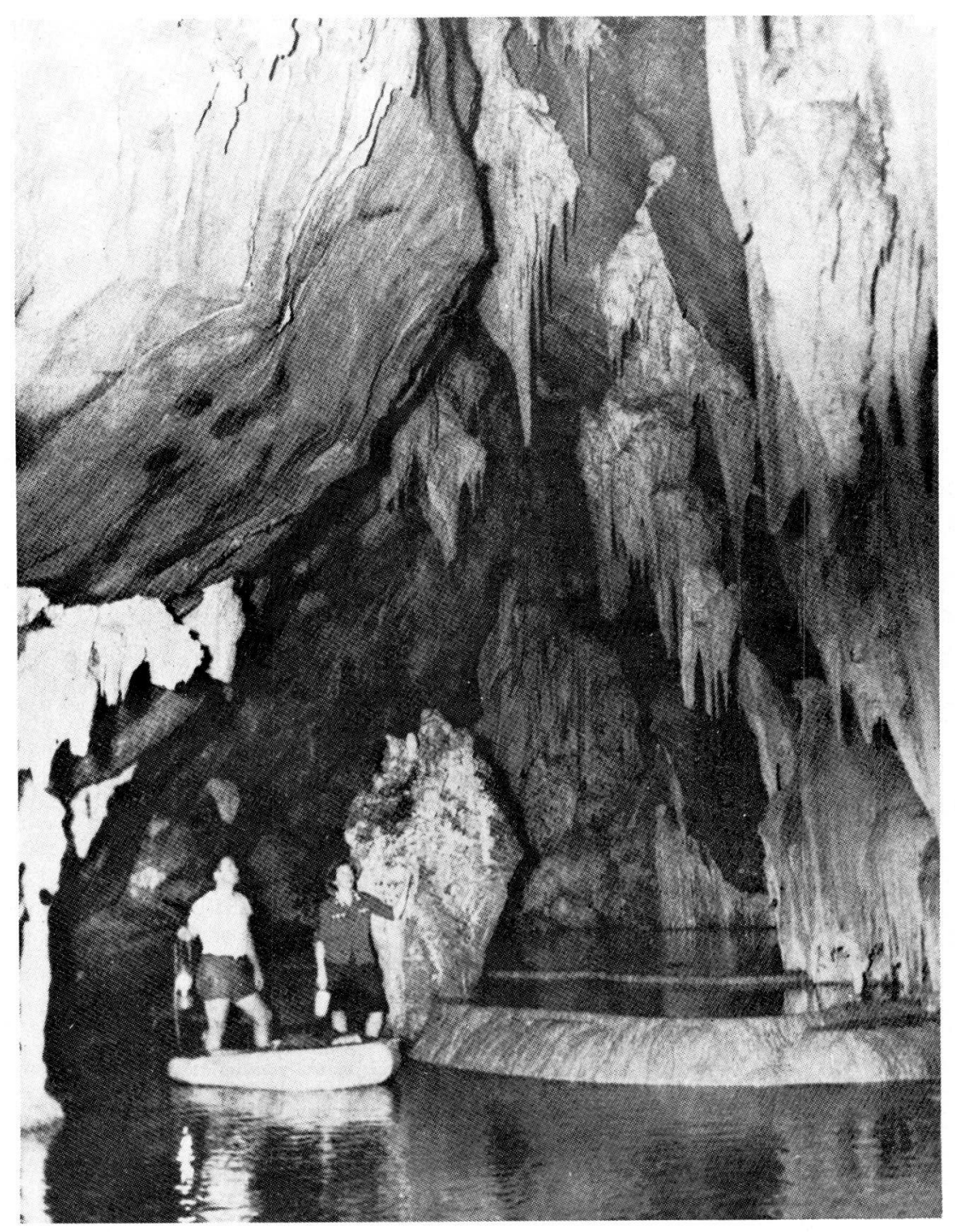

Fig. 1. Le cours souterrain temporaire de la Cueva del Segundo Cauce (Sierra de Quemado), avec ses grands bassins ou "represas" (d'après A.Nuñez Jiménez). 
mentionnons: le Cambariné Procambarus niveus Hobbs \& Villalobos retrouvé dans la localité d'où il avait été décrit (les grandes cuvettes remplies d'eau remanente dans le lit du ruisseau souterrain de la Gran Caverna de Santo Tomás); un très remarquable Triclade dépigmenté et anophthalme, probablement d'origine marine, pris en grand nombre dans les laisses d'eau à fond tapissé d'argile, de la Cueva El Rabon (C'harco Redondo).

Dans Cueva Jibara, j'ai pu mettre en évidence, (probablement pour la première fois dans un pays tropical), l'existence d'une faune interstitielle dans les alluvions d'un ruisseau souterrain (troglorhythrostygal), faune comprenant des Ostracodes, Copépodes, Halacarides etc.

Mais 1'habitat aquatique le plus passionnant qui s'offre aux yeux du biospéologue dans une grotte de Cuba, est celui représenté par les nombreux lacs d'eau douce en surface, mais présentant sans doute une stratification chimique enregistrant des variations et dont l'étude réserve des surprises (températures de l'eau enregistrées par nous en hiver-printemps : $22-25,2^{\circ} \mathrm{C}$ ). Décrits sous le nom de "lagos freaticos", ces lacs que l'on trouve parfois en profondeur dans les grottes, mais parfois à proximité des entrées ou même sous de simples voûtes, à la lumière du jour (dans ces derniers cas il s'agit de cénotes plus ou moins typiques) sont, dans de nombreux cas, des parties constitutives d'énormes nappes de conduits et de fissures (zone noyée du karst) et leurs miroirs correspondent vraiment à la "water table". Grâce aux effort:s des chercheurs cubains et autres, de nombreux éléments troglobies (karstostygobies) de grande taille furent décrits ou simplement signalés des lacs des grottes de Cuba. Il s'agit : d'une Eponge d'eau douce, unique représentant stygobie de ce groupe, découvert par Nuñez-Jiménez mais malheureusement resté non décrit; des Isopodes Cirolanides Troglocirolana cubensis (Hay) et Haptolana trichostoma Bowman ainsi que de l'Anthuride Cyathura specus Bowman; des Amphipodes Weckelia caeca (Weckel) et Paraweckelia silvai Shoemaker; des Décapodes Typhlatya garciai Chace qui est un Atyide, Troglocubanus calcis (Rathbun), T. eigenmanni (Hay), T. gibarensis (Chace) et T. inermis (Chace) - tous des Palaemonides; des Poissons Lucifuga subterraneus Poey et L. dentatus (Poey). Lors de nos recherches nous avons pu retrouver la plupart de ces stygobies de grande taille, dans leurs stations d'origine ou bien dans de nouvelles stations. Mes échantillons contenaient des nouvelles espèces de Mysides, d'Amphipodes et de Décapodes. Les Mlysides sont représentés par Antromysis cubanica Bacescu et Orghidan et Spelaeomysis nuniezi Bacescu et Orghidan; la première de ces espèces, correspondant très probablement à ce que Bolivar avait déjà signalé de la Cueva del Quintanal comme "Antromysis sp." est maintenant connue d'une grotte de la zone de Aston, à la limite des Provinces Pinar del Rio et Habana, et d'une autre de la Peninsula de Guanahacabibes; la seconde fut trouvée dans la même grotte de la zone de Aston mais aussi dans une grotte de l'îlot Caguanes, au $\mathrm{N}$ de la Province Las Villas. Un Amphipode nouveau, Weckelia cubanica Dancau, fut découvert dans deux grottes de lá zone de Aston. Une nouvelle espèce de Décapode du genre Typhlatya ( $T$. consobrina Botosaneanu et Holthuis) est actuellement connue d'une grotte de Sierra de Cubitas et d'une autre de la Peninsula de Guanahacabibes. D'origine marine indiscutable dans la plupart des cas, montrant des degrés différents 


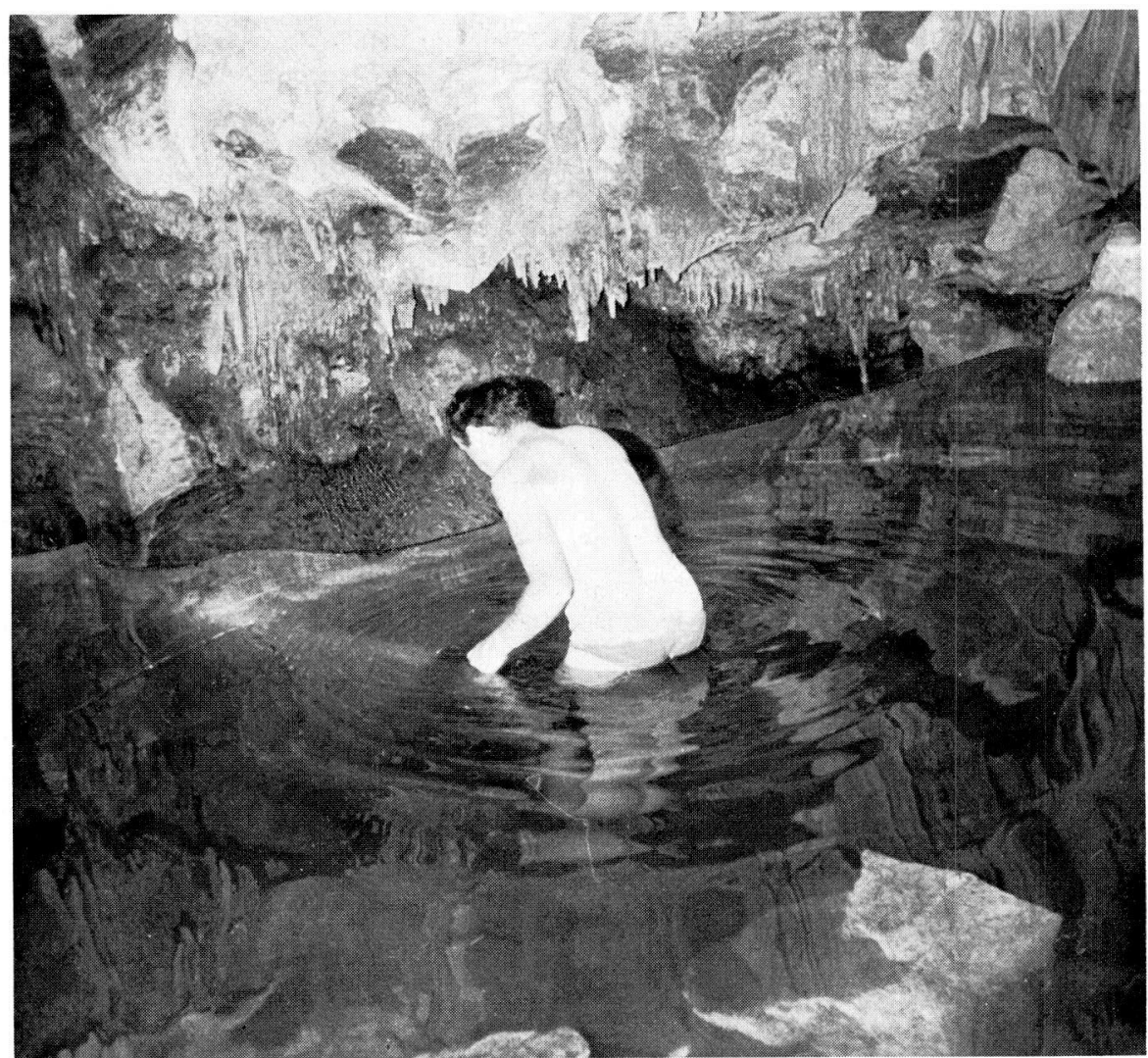

Fig. 2. Pêche de troglobies aquatiques dans un "lago freatico", celui de la Cueva de los Panaderos, près Gibara.

d'adaptation morphologique au milieu souterrain, à distribution généralement limitée et présentant dans de nombreux cas des affinités évidentes avec des troglobies dulçaquicoles des autres îles des Grandes et des Petites Antilles, des Bahamas, du Mexique, de Floride, des Galapagos, les remarquables crustacés et poissons des grottes cubaines sont appelés à jouer un rôle important dans l'élucidation aussi bien des problèmes paléobiogéographiques de la zone antillaise, que des problèmes de l'origine des peuplements souterrains de cette zone.

La faune microscopique des lacs des grottes cubaines n'était pas du tout connue. Nos prélèvements montrent que cette fraction de la faune est particulièrement riche, deux des groupes les mieux représentés étant les Ostracodes et les Copépodes (Cyclopoides et Harpacticoïdes); signalons la présence de Calanoïdes et celle de Cladocères (des éléments épigés sont périodiquement introduits dans ces lacs, et en masse, à la suite des orages). 
La vraie nature hydrologique des lacs des grottes de Cuba n'est pas toujours élucidée. Dans le cas des "grottes du type Aston" - qui sont à vrai dire des cénotes plus ou moins typiques - les lacs sont effectivement les parties accessibles à l'homme de la zone noyée, leurs miroirs correspondent à la "water table", ils méritent pleinement l'appellation de "lagos freaticos". Mais j'ai vu aussi des "lacs" qui ne sont que des laisses de cours d'eau temporaires, d'autres qui sont plutôt des accumulations d'eau de percolation, et dans d'autres cas enfin on ne sait trop que dire. Nous allons évoquer ici brièvement le Lago Marti de Cueva Grande de Caguanes. Cette grotte d'un ilot au $\mathrm{N}$ de la Province Las Villas possède un lac qui représente sans doute, par le pullulement de sa faune, une des stations aquatiques souterraines les plus extraordinaires du globe; Spongiaires (apparemment disparus à présent), Cladocères. Ostracodes, Copépodes Cyclopoïdes et Calanoïdes, Amphipodes comme Paraweckelia silvai et Hyallela azteca (le premier, unique représentant des amphipodes en 1958, avait été apparemment éliminé par le second en 1969), Mysidacés (Spelaecmysis nuniezi), Isopodes Anthurides (Cyathura specus), Décapodes (Troglocubanus gibarensis), font de ce lac une des stations aquatiques souterraines les plus extraordinaires du monde, véritable laboratoire souterrain naturel où des recherches écologiques approfondies donneraient des remarquables résultats.

Il n'y a pas que les grottes qui permettent de jeter des regards vers les eaux circulant dans le karst à Cuba. La faune de quelques puits artificiels a pu être collectée, soit à l'aide de l'appareil de Cvetkov, soit par filtrage de l'eau extraite à l'aide de pompes. Les puits dont j'ai pu collecter la faune sont surtout des cylindres creusés dans la masse des calcaires ou des serpentinites, et qui aboutissent à une nappe d'eau douce ou légèrement saumâtre; à juger d'après le résultat de l'examen d'échantillons peu nombreux, la faune des puits de Cuba est assez riche et variée: Polychètes, Ostracodes, Copépodes, Isopodes Microparasellides, Halacarides, Hydracariens etc., tous ces groupes donnent leur contingent de formes à décrire, tandis que les éléments de taille considérable (Cirolanides, Décapodes, Poissons) sont rarement présents dans les nappes accessibles par ces puits à l'encontre de ce que l'on sait des puits du Texas ou de ceux du Mexique.

Une carte du karst de Cuba montre l'énorme extension du karst sous-marin tout autour de l'ile. Ce karst submergé est inaccessible au biospéologue ignorant des méthodes de la plongée sous-marine, mais on peut se faire une idée du peuplement de cette extraordinaire "zone tampon" entre le domaine marin et celui des eaux douces du karst, en explorant les "grietas", fentes verticales peu profondes dans les calcaires des terrasses marines au voisinage du littoral, ou les "casimbas", sortes de puits naturels peu profonds creussés dans ces mêmes calcaires; plus ou moins éclairées, ces grietas et casimbas donnent accès à une eau fortement saumâtre en saison sèche mais dont la teneur en sels est sûrement fort amoindrie après les pluies d'orage; c'est l'habitat typique du Décapode Hyppolitide Barbouria cubensis (von Martens) connu seulement de Cuba et de l'ile Gran Cayman, animal oculé d'une belle couleur rouge, qui est sans doute en voie de coloniser le domaine souterrain justement par l'intermédiaire de l'immense réseau de fentes dont les "grietas" et "casimbas" sont seules abordables pour l'explorateur. Barbouria est accompagné 


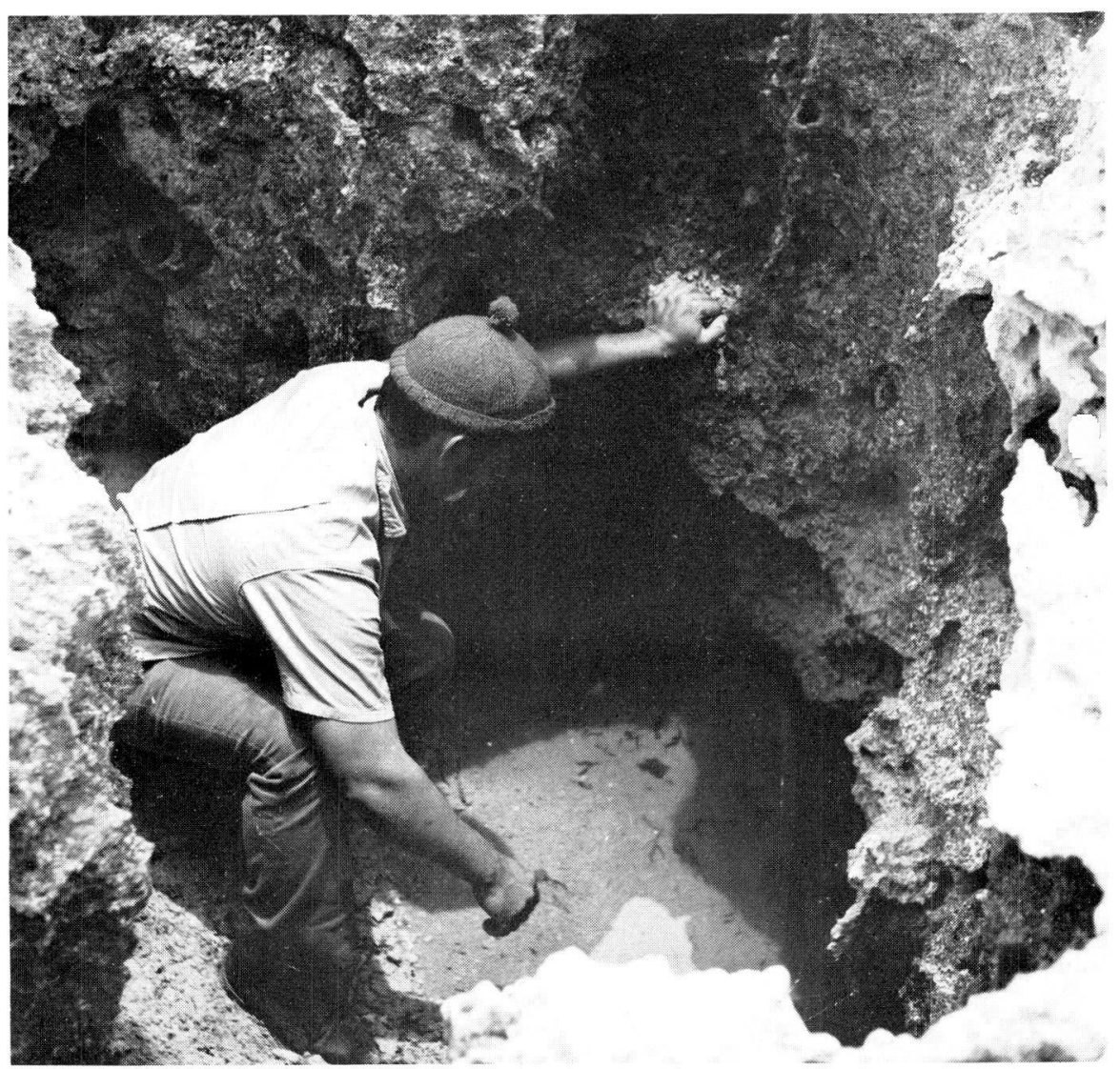

Fig. 3. Une "casimba" partiellement remplie d'eau, dans les calcaires quaternaires du S. de Isla de Pinos.

dans les grietas par des crustacés microscopiques absolument remarquables, tels l'Ostracode Thaumatocypris orghidani Danielopol (seconde espèce d'un genre qui n'était auparavant connu que par une espèce marine abyssale).

Je n'ai jamais eu l'occasion de faire des observations sur la faune des puits creusés dans les alluvions des grandes vallées, puits permettant donc de jeter un regard sur les vraies nappes phréatiques (eustygal). C'est une lacune à combler. En effet, il serait fort instructif de pouvoir comparer à Cuba les peuplement de la zone noyée des karsts et ceux des nappes phréatiques proprement dites.

Les milieux interstitiels de Cuba - et des Antilles en général - restaient vierges de toute recherche. J'ai pu faire d'assez nombreux sondages dans les plages marines (thalassostygal), aussi bien dans celles de la Mer de Caraïbes que dans celles de l'Océan Atlantique; les résultats ont été parfois excellents, parfois décevants; ce fut 
l'occasion de faire des observations sur l'extrême importance du facteur granulométrique dans la répartition de la faune dans le milieu interstitiel littoral (faune plus riche et variée darıs les sables d'origine fluviatile et meubles que dans les sables coralliens, tassés ou à interstices remplis par du détritus végétal). L'étude de plusieurs groupes a déjà fourni les premiers documents sur la faune mésopsammale des Grandes Antilles, tandis que d'autres groupes (Nématodes, Harpacticoïdes, Tanaidacés, Décapodes, Diploures, Collemboles) sont à l'étude. Les Polychètes interstitiels marins de nos récoltes, étudiés par $\mathrm{M}^{\mathrm{me}}$ Hartmann Schröder, appartiennent aux genres Hesionides, Eusyllis, Sphaerosyllis, Typosyllis (avec la nouvelle espèce $T$. botosaneanui Hartmann Schröder) et Namanereis; un seul Cyclopoïde (genre Neocyclops) a été décrit jusqu'à présent; l'Ostracode Pussella botosaneanui Danielopol est l'unique représentant connu de la nouvelle famille des Pussellides; ce sont les Isopodes Microparasellides et Microcerberides qui ont fourni le contingent le plus important de nouveautés (une espèce de Microcharon, genre signallé pour la première fois des Amériques; une espèce de Angeliera, même situation; trois espèces de Microcerberus; un genre nouveau, Yvesia Coineau et Botosaneanu, qui est apparanté à Microcerberus; enfin, une espèce fort petite de Cyathura); parmi les Acariens, les Halacarides sont représentés par le bizarre Halacarus anomalus et les Rhodacarides ont permis la description par Beron et Petrova de deux nouvelles espèces de Rhodacarus.

Avec ses eaux courantes soit intermittentes, traversant des zones calcaires, soit charriant beaucoup de limon qui vient colmater les interstices des graviers roulés,

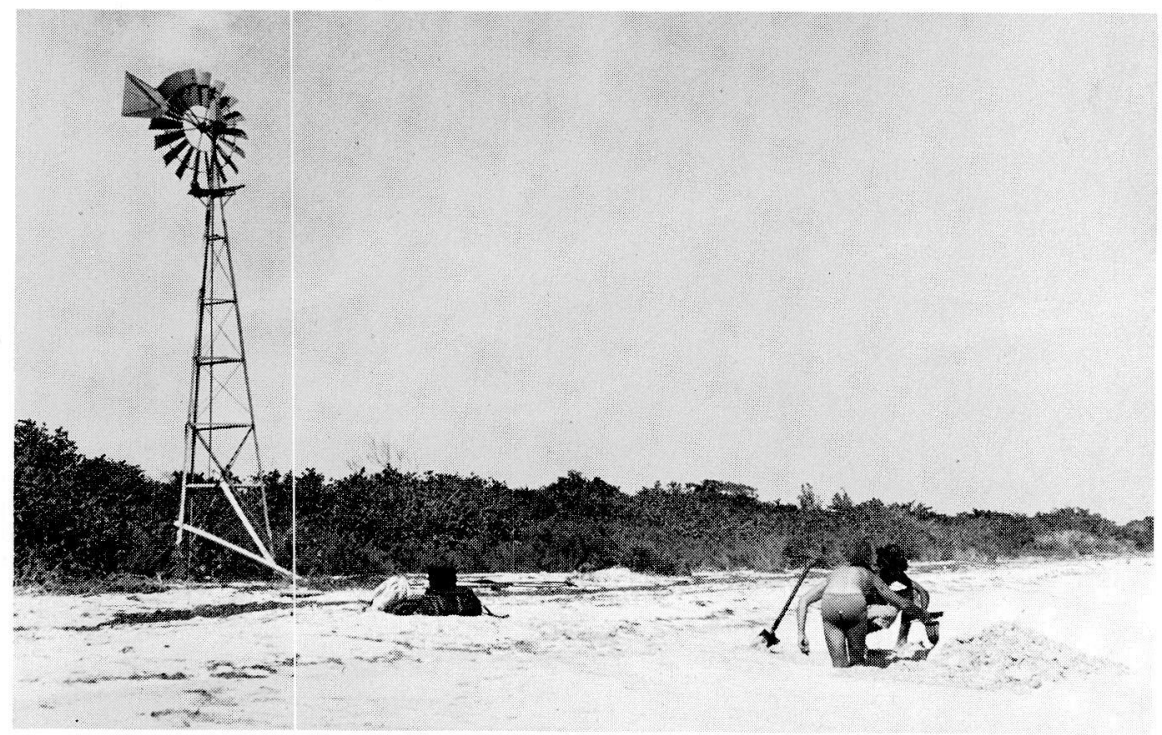

Fig. 4. Sondage dans le plage de Punta del Este (I. de Pinos) pour la récolte de la faune interstiticlle; une pompe à "molino de viento" (à gauche) extrait une eau phréatique saumâtre. 
Cuba n'est pas un pays très propice pour des recherches sur la faune des sous-écoulements fluviatiles (rhythrostygal, milieu hyporhéique). Cependant, des endroits favorables peuvent être trouvés dans les montagnes cristallines, et les sondages Karaman-Chappuis que j'ai pu faire surtout dans les M-ts Sagua-Baracoa ou au pied de la Sierra Cristal (Prov. Oriente) ont démontré sans conteste l'existence sous les tropiques d'une faune interstitielle dulçaquicole parfois riche et variée, dont il faut citer les nombreux Ostracodes et Amphipodes, les Isopodes microscopiques (Microcharon phreaticus Coineau \& Botosaneanu, Cyathura sp.), les Hydracariens représentés par des éléments assez variés, les Polychètes, les Turbellariés etc. Mentionnons, comme élément négatif non dépourvu d’importance, l'absence des Syncarides, jamais découverts en Amérique du Nord et Centrale mais fort bien représentés en Amérique du Sud.

Des recherches biospéologiques sont actuellement en cours un peu partout en Amérique Centrale, qui non seulement font ressortir l'importance exceptionnelle de

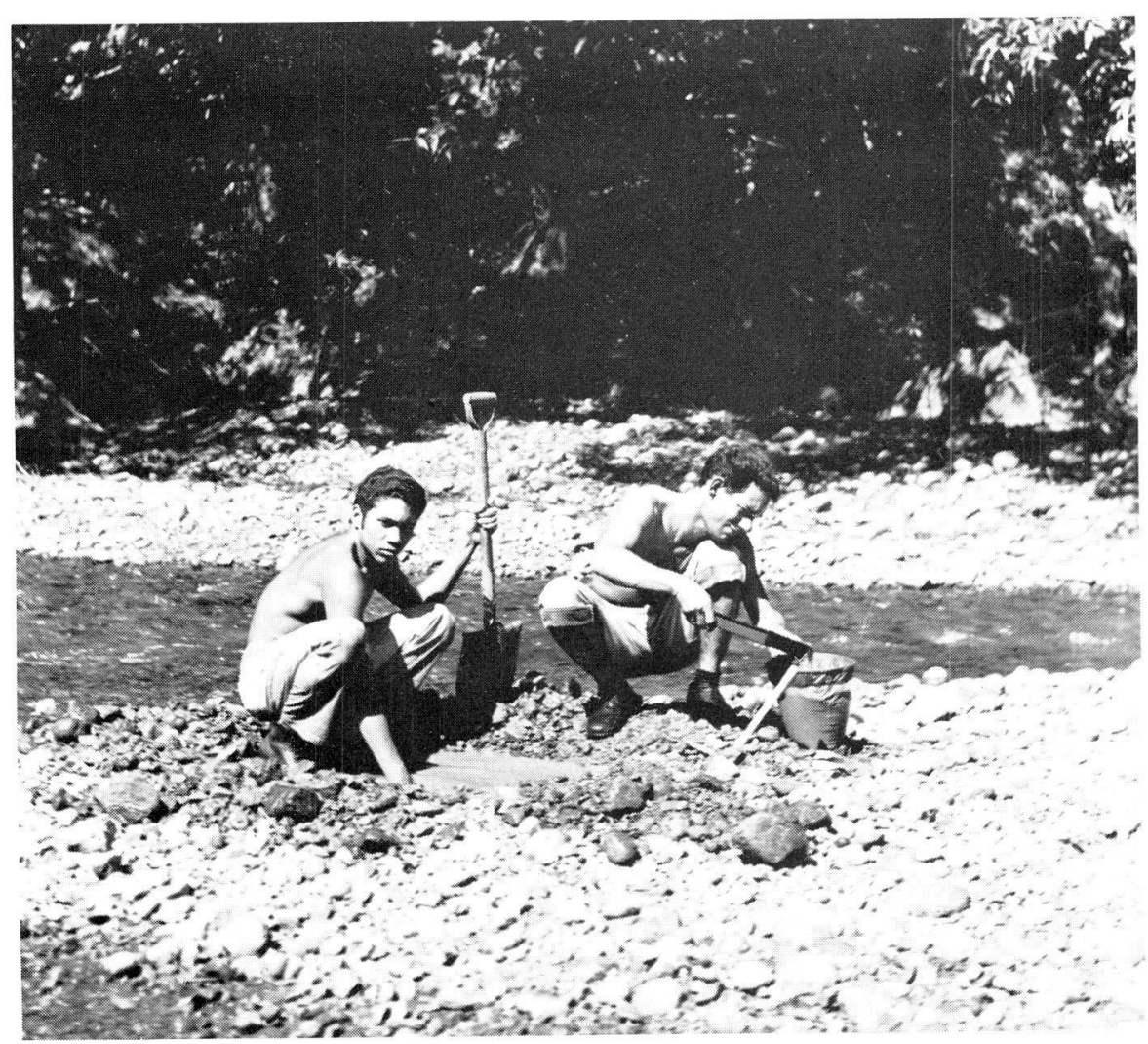

Fig. 5. A la recherche de la faune des sous-écoulements fluviatiles: Rio Sabanilla près Baracoa. 
la région pour notre domaine, mais qui permettront sous peu de la considérer comme une des mieux connues. Le Mexique est systématiquement fouillé depuis des années par les chercheurs des U.S.A., auxquels viennent s'ajouter des missions italiennes et allemandes; une assez vive activité due à des chercheurs de plusieurs nationalités se déroule à Guatemala, à Panama, dans la plupart des Grandes et des Petites Antilles, ainsi qu'aux Bahamas (et aux Galapagos). Les expéditions biospéologiques cubano-roumaines à Cuba ont pour objectif de combler les lacunes les plus importantes qui subsistent dans la connaissance de la faune souterraine de la plus grande des iles antillaises. Parmi les résultats obtenus ou à entrevoir, mentionnons ceux concernant la biogéographie. En efudiant un important groupe de la faune hypogée terrestre de Cuba, les Isopodes Oniscoïdes, Vandel (1972) arrive à la conclusion que "la totalité des espèces ... représentent des rélictes d'origine sud-américaine" et que "nous sommes conduits à interpréter l'île de Cuba - et probablement les autres constituants des Grandes Antilles - comme une terre qui s'est détachée de l'Amérique du Sud ..."; la comparaison des faunes aquatiques souterraines de Cuba avec celles des terres voisines, conduit parfois à une conclusion qui corrobore celle de Vandel mais parfois (par exemple dans le cas de certains éléments d'origine marine) à une conclusion radicalement différente: nette parenté avec la faune du Mexique par exemple; il faudra élucider les causes de cette apparente contradiction.

Mais les résultats écologiques promettent, eux aussi, d'être intéressants. Nos recherches apporteront une contribution au problème des sous-divisions du domaine aquatique souterrain dans les conditions d'un climat chaud et humide, en précisant la manière dont s'articulent entre elles ces sous-divisions. Ensuite ces recherches permettront de compléter la réponse récemment donnée par R. Mitchell à un des problèmes majeurs de la biospéologie : celui des différences entre les communautés cavernicoles des zones tempérées et tropicales, et des causes de ces différences. Enfin -last but not least- c'est sous ses aspects les plus typiques et en même temps les plus variés que pourra être surpris ici, dans son déroulement même, le passionnant processus de la colonisation du domaine aquatique souterrain par des éléments originaires de la Mer et ayant utilisé comme voie de pénétration, soit le domaine interstitiel, soit celui des fentes et fissures des calcaires littoraux.

\section{RESUME}

Bref compte-rendu sur quelques résultats obtenus par les expéditions biospéologiques cubano-rounaines à Cuba dans l'étude des faunes aquatiques souterraines. Les divisions suivantes du domaine aquatique souterrain sont passées en revue en même temps que les éléments les plus caractéristiques de leur faune: bassins à dépôt de guano sur le fond, ainsi que gours véritables, dans la zone vadose des grottes; cours d'eau souterrains; lacs ("phréatiques" et autres) dans les grottes; puits creusés dans les calcaires, et "grietas" - fentes verticales dans les calcaires des terrasses marines, donnant accès à une eau douce ou saumâtre -; interstitiel des plages marines; sous-écoulement des cours d'eau. 
Actuellement, des recherches biospéologiques sont en cours un peu partout en Amérique Centrale; Cuba restait, récemment encore, peu étudié et on se rend compte du fait que c'est une des zones les plus remaquables à ce point de vue. On donne une enumération de quelques-uns des problèmes les plus intéressants que soulève l'étude de la faune aquatique souterraine de Cuba. Intéressant problème biogéographique: certains éléments aquatiques souterrains s'avèrent être étroitement apparentés à des éléments appartenant à la faune des autres Antilles (et du Mexique), mais non à la faune sud-américaine (comme c'est le cas pour quelques groupes terrestres). Les recherches vont apporter une contribution au problème des divisions du domaine souterrain aquatique, des relations entre ces divisions, sous un climat chaud et humide; elles permettront peut-être de donner une solution du problème des différences entre communautés cavernicoles des grottes tempérées et tropicales; enfin, ces recherches permettront de suivre dans son déroulement même le processus de colonisation des eaux douces souterraines par des éléments d'origine marine, soit par l'intermédiaire du domaine interstitiel, soit par les fissures des calcaires littoraux.

\section{SUMMARY}

A short account on some achievements of the cubano-romanian biospeleological expeditions to Cuba in the study of the aquatic subterranean faunas. The following divisions of the aquatic subterranean realm are reviewed together with their most characteristic faunal elements: "guano pools" and rimstone pools in the vadose zone of the caves; underground streams; water table (and other) lakes in the caves; "pozzos" carved in the limestone, and "grietas" which are vertical clefts in the limestone of marine terrasses, giving access to fresh- or to brackish water; the interstitial of the marine beaches; the underflow of running waters.

At present, thorough biospeleological research is being carried out almost everywhere in Central America; Cuba, which remained until recently rather poorly investigated, proves to be one of the most remarkable areas from this point of view. A few of the most interesting problems raised in the course of the study of the underground aquatic fauna of Cuba are listed. An interesting biogeographical problem is the following: some of the subterranean aquatic elements prove to be related to elements belonging to the fauna of the other Antilles and of Mexico, but not to the South-American fauna (as is the case for some terrestrial groups). The research undertaken will be a contribution to the problem of the divisions of the aquatic subterranean realm and of their reciprocal relations, in a warm and humid climate; it will also contribute an answer to the problem of the differences between temperate and tropical cave communities; finally, it allows one to perceive in its very progress the process of colonization of the subterranean freshwaters by elements of marine origin, either through the interstitial realm or through the fissures of the littoral limestones.

\section{BIBLIOGRAPHIE}

Résultats des expéditions biospéologiques cubano-roumaines à Cuba. Vol. 1. pp.1-424. Editura Academici R.S.R., Bucarest, 30 aôut, 1973. 